\title{
结合面静摩擦因数尺度关联三维分形模型*
}

\author{
张学良 张 伟 温淑花 姚世生 \\ (太原科技大学机械工程学院 太原 030024 )
}

\begin{abstract}
摘要: 针对现有结合面静摩擦因数分形模型的静摩擦因数随结合面法向接触载荷增大而增大, 与试验研究结论及统计模型不 一致的问题, 基于尺度等级定义微凸体的大小, 严格区分微凸体高度与变形, 构建各尺度等级微凸体的法向接触载荷与接触 面积之间关系及其发生弹性和弹塑性第一变形时所能承受的最大切向载荷即最大静摩擦力计算模型, 进而建立结合面法向接 触载荷与最大静摩擦力计算模型, 在此基础上, 依据结合面静摩擦因数定义, 提出与微凸体尺度等级关联的考虑微凸体完全 弹性、弹塑性和完全塑性三种变形机制的结合面静摩擦因数三维分形模型, 数值仿真分析了结合面静摩擦因数与法向接触载 荷和分形维数 $D$ 等的关系, 结果表明结合面静摩擦因数随着结合面法向接触载荷的增大而减小, 随着分形维数的增大而增大, 并试验实例验证了所建模型的正确性, 解决了现有结合面静摩擦因数分形模型与统计模型和试验结果之间的不一致性。
\end{abstract}

关键词: 结合面; 静摩擦因数; 尺度关联; 分形模型

中图分类号: TH113

\section{Three-dimensional Fractal Model with Scale Correlation for Static Friction Factor of Joint Interfaces}

\author{
ZHANG Xueliang ZHANG Wei WEN Shuhua YAO Shisheng \\ (School of Mechanical Engineering, Taiyuan University of Science and Technology, Taiyuan 030024)
}

\begin{abstract}
In view of the fact that the static friction coefficient of the fractal model of the static friction coefficient of the existing interface increases with the increase of the normal contact load of the interface, which is inconsistent with the experimental results and the statistical model. The size of the micro convex body is defined based on the scale level, and the height and deformation of the micro convex body are strictly distinguished. The relationship between the normal contact load and the contact area of the micro convex body at each scale level and the calculation model of the maximum tangential load that the micro convex body can bear when the full elastic and the first elastic-plastic deformation occur are established. Finally, the calculation model of the relationship between the normal contact load and the maximum static friction force is established. On this basis, according to the definition of the static friction coefficient of the joint surface, a three-dimensional fractal model of the static friction coefficient of the joint surface is proposed, which is related to the scale level of the micro convex body and considers three deformation mechanisms of the micro convex body, i.e. complete elasticity, elastoplasticity and complete plasticity. The relationship between the static friction coefficient of the joint surface and the normal contact load and fractal dimension $D$ is analyzed by numerical simulation. The results show that the static friction coefficient decreases with the increase of normal contact load and increases with the increase of fractal dimension. By comparing with the experiment, the correctness of the model is proved, and the inconsistency between the fractal model of static friction coefficient and the statistical model and the experimental results is solved.
\end{abstract}

Key words: joint interfaces; static friction factor; scale correlation; fractal model

\section{0 前言}

摩擦是一种自然现象, 任何物体接触表面都存

* 国家自然科学基金(51275328)、山西省自然科学基金(201901D111248) 和山西省 “1331” 工程重点学科建设资助项目。20200502 收到初稿, 20201210 收到修改稿
在着摩擦, 而有摩擦必然会产生能量消耗和表面材 料的磨损，据估计全球有 1/3 1/2 左右的各类能源 被摩擦消耗掉, 研究和预测机械系统的摩擦是具有 重要意义。静摩擦因数是摩擦学重要参数之一, 长 期以来人们对机械结合面静摩擦因数开展了许多研 究工作，传统的库仑摩擦定律认为结合面静摩擦因 
数仅随接触表面材料的不同而变化, 而与所施加的 法向载荷无关。随着摩擦学的不断发展和现代科技 的进步, 已有试验研究发现结合面静摩擦因数对于 同种材料不变的结论与实际情况不符, 而是随着接 触压力的增加而减小 ${ }^{[1-2]}$ 。目前结合面静摩擦因数计 算模型主要有如下两类。

(1) 结合面静摩擦因数统计模型。

1988 年 $\mathrm{CHANG}$ 等 ${ }^{[1]}$ 在 GW 模型的基础上, 基 于弹性接触微凸体承受切向载荷的能力, 建立了结 合面静摩擦因数统计模型(简称为 CEB 摩擦模型)。

KOGUT 和 ETSION 等 ${ }^{[2-3]}$ 研究发现除了弹性接触微 凸体, 弹塑性接触微凸体也具有承受切向载荷的能 力, 因而 CEB 摩擦模型低估了结合面静摩擦因数。 尤晋闽等 ${ }^{[4-5]}$ 在考虑微凸体完全弹性、弹塑性和完全 塑性变形的基础上, 进一步研究建立了结合面静摩 擦因数统计模型。

(2) 结合面静摩擦因数分形模型。

盛选禹等 ${ }^{[6]}$ 在 MB 分形模型的基础上, 建立了 结合面静摩擦因数分形模型。在此基础上, 田红 亮等 ${ }^{[7-8]}$ 对结合面分形理论进行改进, 进一步研究建 立了结合面静摩擦因数分形模型。

然而以上两类模型计算得出的结合面静摩擦因 数随结合面法向无量纲接触载荷的变化规律却截然 相反。结合面静摩擦因数分形模型的静摩擦因数随 着结合面无量纲法向接触载荷的增加而增大, 与文 献[9-10]的试验结论相反, 而结合面静摩擦因数统计 模型的静摩擦因数却随着结合面无量纲法向接触载 荷的增加而减小, 与文献[9-10]的试验结果相同。导 致这两种模型结果不同的最根本原因在于 MB 分形 理论认为微凸体变形与所受载荷大小无关, 是完全 变形, 即微凸体高度就是微凸体变形, 与经典接触 力学相矛盾, 同时 $\mathrm{MB}$ 分形理论认为微凸体峰顶曲 率半径 $R$ 是一个与接触面积有关的参数, 而不是一 个定值, 据此得出了微凸体先发生完全塑性变形再 发生弹塑性变形和弹性变形的结论, 而在 GW 模型 中微凸体的平均峰顶曲率半径 $R$ 是一个确定的参 数, 建立在此基础之上的微凸体变形过程为先发生 弹性变形再发生弹塑性变形进而发生完全塑性变 形, 且在该模型基础之上建立的结合面静摩擦因数 统计模型的预测结果也与试验相符。然而统计学参 数受测量仪器分辨率与测量条件的影响, 导致对于 确定的粗䊁表面其轮廓统计参数不是唯一的, 这就 使得以这些参数值为基础建立的结合面静摩擦因数 统计模型的预测结果也不是唯一的 ${ }^{[11]}$ 。尽管结合面 分形理论模型能够利用分形参数描述所有尺度范围
内的全部粗糙度信息, 但由于 MB 模型自身的缺陷 导致了在其基础上建立的结合面静摩擦因数分形模 型预测结果的一些不合理性, 且以上分形模型大多 是建立在二维分形模型的基础之上, 用二维分形模 型计算三维结合面的接触特性存在着理论上的不合 理性 ${ }^{[12]}$ 。

针对上述问题, 本文将在前人研究工作的基础 上, 综合考虑结合面微凸体弹性、弹塑性和塑性三 种变形机制, 研究建立结合面静摩擦因数尺度关联 三维分形模型。首先基于尺度等级定义微凸体的大 小, 严格区分微凸体高度与变形, 解决 MB 分形模 型与经典接触力学之间的矛盾, 建立各尺度等级的 微凸体在法向接触载荷作用下发生弹性和弹塑性第 一变形时所能够承受的最大切向载荷即最大静摩擦 力, 进而基于结合面静摩擦因数定义, 建立结合面 静摩擦因数尺度关联三维分形模型, 解决现有结合 面静摩擦因数分形模型与统计模型和试验结果之间 的不一致性。

\section{1 结合面静摩擦因数尺度关联三维分 形模型}

\section{1 粗粮表面三维分形数学表征}

粗糙表面具有统计自仿射性和多尺度的分形特 征, 研究发现这种特征可以用具有双变量的 WM 函 数表征 ${ }^{[12-13]}$

$$
\begin{gathered}
Z(x, y)=L\left(\frac{G}{L}\right)^{(D-2)}\left(\frac{\ln \gamma}{M}\right)^{0.5} \sum_{m=1}^{M} \sum_{n=0}^{n_{\max }} \gamma^{(D-3) n} \times \\
\left\{\cos \varphi_{m, n}-\cos \left[\frac{2 \pi \gamma^{n}\left(x^{2}+y^{2}\right)^{0.5}}{L} \times\right.\right. \\
\left.\left.\cos \left(\arctan \left(\frac{y}{x}\right)-\frac{\pi m}{M}\right)+\varphi_{m, n}\right]\right\} \\
n_{\max }=\operatorname{int}\left[\frac{\ln \left(L / L_{S}\right)}{\ln \gamma}\right]
\end{gathered}
$$

式中, $Z(x, y)$ 为轮廓高度; $x, y$ 为轮廓的位移坐标; $\gamma$ 为随机轮廓的空间频率, 基于表面粗䊁度和频谱 分布密度考虑, 取 $\gamma=1.5 ; n$ 为频率指数, 表示微凸 体的尺度等级; $M$ 为构造表面重叠隆起的个数; $n_{\text {max }}$ 为与距离截止长度有关的频率指数上限; $L$ 为 分形采样长度; $L_{s}$ 为最低截至长度, 本文中取值与 文献[13]相同即 $L_{s}=10^{-9} \mathrm{~m} ; \varphi_{m, n}$ 为随机相位; 为分 形特征长度尺度参数。 
当 $M=1, m=1$ 式(1)变为单自变量的 $\mathrm{WM}$ 函 数 ${ }^{[12]}$

$$
\begin{aligned}
& z(x)=L\left(\frac{G}{L}\right)^{D-2} \sqrt{\ln \gamma} \sum_{n=0}^{n_{\max }} \gamma^{n(D-3)} \times \\
& {\left[\cos \varphi_{1, n}-\cos \left(\frac{2 \pi \gamma^{n} x}{L}-\varphi_{1, n}\right)\right]}
\end{aligned}
$$

\section{2 粗粘表面微凸体的尺度等级、几何特征表征与 接触变形}

本文采用与 $\mathrm{MB}$ 模型相同的假设, 将结合面等 效为一个理想光滑刚性平面和一个分形粗糙面的接 触 ${ }^{[14]}$, 单个微凸体的接触变形示意图如图 1 所示。 在刚性平面施加一法向载荷时, 尺度等级为 $n$ 的微 凸体将产生法向变形 $\omega_{n}, \delta_{n}$ 为尺度等级 $n$ 的微凸体 的高度, $\omega_{n}$ 不同于 $\delta_{n}, R_{n}$ 为尺度等级 $n$ 的微凸体 的峰顶曲率半径, $2 r_{t n}$ 为尺度等级 $n$ 的微凸体真实接 触长度, $2 r_{n}$ 为尺度等级 $n$ 的微凸体的截断长度, $2 r_{n}^{\prime}$ 为尺度等级 $n$ 的微凸体的基底长度, 若取样长 度 $L=2 r_{n}^{\prime} \gamma^{n}$, 则此取样长度对应的微凸体尺度等级 为 $n=\ln \left(L / 2 r_{n}^{\prime}\right) / \ln \gamma$, 令随机相位 $\phi_{m, n}=0$, 则尺 度等级为 $n$ 的微凸体变形前的轮廓曲线为 ${ }^{[15-16]}$

$$
z_{n}(x)=G^{D-2}(\ln \gamma)^{0.5}\left(2 r_{n}^{\prime}\right)^{(D-3) n} \cos \left(\frac{\pi x}{r_{n}^{\prime}}\right)
$$

其峰顶曲率半径为 ${ }^{[13]}$

$$
R_{n}=\frac{\left[L /\left(2 \gamma^{n}\right)\right]^{D-1}}{2^{3-D} \pi^{2} G^{D-2}(\ln \gamma)^{0.5}}
$$

微凸体的高度

$$
\delta_{n}=G^{(D-2)}(\ln \gamma)^{1 / 2}\left(\frac{L}{\gamma^{n}}\right)^{(3-D)}
$$

可见, $R_{n}$ 与微凸体尺度等级 $n$ 有关, 微凸体尺度等 级 $n$ 确定后, $R_{n}$ 就是确定的, 与其接触变形或接触 面积无关。

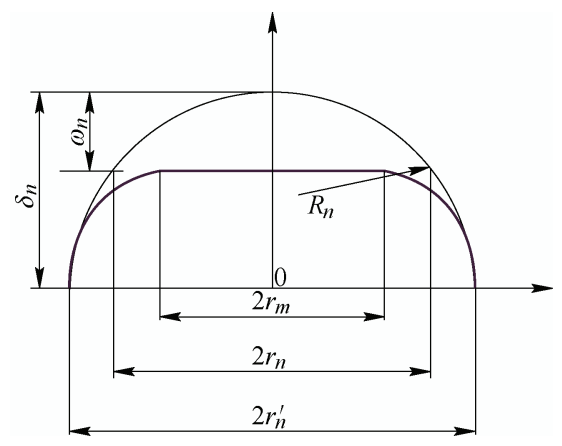

图 1 单个微凸体接触变形示意图

\section{3 微凸体所能承受的最大切向载荷}

尺度等级为 $n$ 的微凸体法向变形量 $\omega_{n}$ 在不同 的范围时, 可将微凸体的接触变形过程分为完全弹 性接触变形阶段、弹塑性接触变形阶段和完全塑性 接触变形阶段, 而由弹性接触变形向弹塑性接触变 形转变的临界弹性接触变形量可用 $\omega_{n e c}$ 表示, 由弹 塑性接触变形向完全塑性接触变形转变的临界接触 变形量可用 $\omega_{n p c}$ 表示, 研究表明 ${ }^{[14]} \omega_{n p c}=110 \omega_{n e c}$ 。

由赫兹接触理论可知尺度等级为 $n$ 的微凸体临 界弹性接触变形量为 ${ }^{[14]}$

$$
\omega_{n e c}=\left(\frac{\pi K H}{2 E}\right)^{2} R_{n}
$$

式中, $K$ 为硬度系数; 与材料的泊松比 $v$ 有关, $K=0.454+0.41 v$ 。 $E$ 为等效弹性模量; $H$ 为较软 材料的硬度。

当尺度等级 $n$ 的微凸体接触变形量范围在 $\omega_{n e c}<\omega_{n}<110 \omega_{n e c}$ 时, 那么微凸体接触变形混合着 弹性接触变形和塑性接触变形, 在初始屈服临界点 $\omega_{\text {nec }}$ 附近以弹性接触变形为主导, 而在塑性临界屈 服点 $110 \omega_{n e c}$ 附近以塑性接触变形为主导。弹塑性接 触变形区域可进一步划分为两个区域, 即弹塑性第 一接触变形区域 $\left(\omega_{n e c}<\omega_{n} \leqslant 6 \omega_{n e c}\right)$ 和弹塑性第二接 触变形区域 $\left(6 \omega_{\text {nec }}<\omega_{n} \leqslant 110 \omega_{n e c}\right)$ 。

根据文献 [5-8]可知在法向接触载荷下只有处于 弹性接触变形状态和弹塑性第一接触变形状态的微 凸体可以承受切向载荷, 故而在计算结合面静摩擦 力时只有处于这两种接触变形状态的微凸体对结合 面静摩擦力有贡献。当接触变形微凸体所承受的切 向载荷不断增大时, 其完全弹性接触变形区和弹塑 性第一接触变形区的接触界面最终达到完全屈服, 此时的最大切向载荷即为其最大静摩擦力。

本文模型采用文献[4-5]的假设, 微凸体接触界 面的屈服发生在接触点的边缘, 而边缘上的应力为

$$
\begin{gathered}
\sigma_{x}=\frac{(1-2 v)}{2 \pi r^{2}} F+\frac{3}{4 r^{2}} T\left(\frac{v}{4}+1\right) \\
\sigma_{y}=\frac{9 v}{16 r^{2}} T-\frac{1-2 v}{2 \pi r^{2}} F \\
\sigma_{z}=\tau_{x y}=\tau_{x z}=\tau_{y z}=0
\end{gathered}
$$

式中, $\sigma_{x}$ 为与正向载荷方向相同的应力; $\sigma_{y}$ 为与 摩擦力方向相同的应力; $\sigma_{z}$ 为垂直于正向载荷和法 向载荷所在平面的力; $F$ 为单个微凸体上的法向接 触载荷。

采用 Tresca 屈服条件 


$$
\begin{aligned}
& \left|\sigma_{1}-\sigma_{2}\right| \leqslant \sigma_{s} \\
& \left|\sigma_{2}-\sigma_{3}\right| \leqslant \sigma_{s} \\
& \left|\sigma_{1}-\sigma_{3}\right| \leqslant \sigma_{s}
\end{aligned}
$$

式中, $\sigma_{s}$ 为较软材料的屈服强度。

由式(6) (8)的应力方程可得

$$
\sigma_{1}=\sigma_{x} \quad \sigma_{2}=\sigma_{y} \quad \sigma_{3}=0
$$

将此代入屈服条件得到当微凸体接触界面达到 完全屈服时, 其所能承受的最大切向载荷即最大静 摩擦力为

$$
T=\frac{8 a \sigma_{s}}{\pi(6-3 v)}+\frac{8(2 v-1)}{\pi(6-3 v)} F
$$

\section{4 微凸体弹性接触变形模型}

当尺度等级 $n$ 的微凸体的高度小于该等级下的 临界弹性接触变形量 $\omega_{n e c}$ 即 $\max \left(\omega_{n}\right)=\delta_{n}<\omega_{n e c}$ 时, 该尺度等级的微凸体只能发生弹性变形。由文献[13] 可知微凸体的弹性临界等级为

$$
n_{e c}=\operatorname{int}\left(\frac{1}{2(D-2) \ln \gamma} \ln \left\{\frac{\left(\frac{K \varphi}{2}\right)^{2} L^{(2 D-4)}}{4 G^{2 D-4} \ln \gamma}\right\}\right)
$$

式中, $\varphi=\frac{H}{E}$; 取样长度取 $L=1 \mathrm{~mm}^{[13]}$ 。

根据赫兹接触理论, 尺度等级为 $n$ (即微凸体 轮廓的频率指数为 $n)$ 的微凸体在弹性接触变形状 态的法向接触载荷和接触面积分别为

$$
\begin{aligned}
& F_{n e}=\frac{4}{3} E R_{n}^{0.5} \omega_{n}^{1.5} \\
& a_{n e}=\pi R_{n} \omega_{n}
\end{aligned}
$$

联立式(4)、(12)和(13)可知尺度等级为 $n$ 的微凸 体发生完全弹性接触变形时, 其法向接触载荷和接 触面积的关系为

$$
F_{n e}=\frac{16 E \pi^{0.5} G^{D-2} \ln (\gamma)^{0.5}}{3 L^{D-1}} a_{n e} e^{1.5} \gamma^{n(D-1)}
$$

从上式可以看出, 完全弹性接触变形状态的单 个微凸体所受法向接触载荷和接触面积的 $3 / 2$ 次方 成正比。

\section{5 微凸体弹塑性接触变形模型}

当尺度等级为 $n$ 的微凸体的接触变形量 $\omega_{n}$ 满 足 $\omega_{\text {nec }}<\omega_{n} \leqslant 6 \omega_{\text {nec }}$ 时, 则其处于弹塑性第一接触变 形状态, 当 $6 \omega_{n e c}<\omega_{n} \leqslant 110 \omega_{n e c}$ 时, 则其处于弹塑 性第二接触变形状态。类似地, 微凸体弹塑性第一 接触变形临界等级和弹塑性第二接触变形临界等级
分别为 ${ }^{[13]}$

$$
\begin{aligned}
& n_{e p c}=\operatorname{int}\left(\frac{1}{2(D-2) \ln \gamma} \ln \left\{\frac{6\left(\frac{K \varphi}{2}\right)^{2} L^{(2 D-4)}}{4 G^{2 D-4} \ln \gamma}\right\}\right) \\
& n_{p c}=\operatorname{int}\left(\frac{1}{2(D-2) \ln \gamma} \ln \left\{\frac{110\left(\frac{K \varphi}{2}\right)^{2} L^{(2 D-4)}}{4 G^{2 D-4} \ln \gamma}\right\}\right)
\end{aligned}
$$

尺度等级 $n$ 的微凸体处于弹塑性第一和第二接 触变形状态的法向接触载荷和接触面积的关系分别 为 $^{[13]}$

$$
\begin{aligned}
F_{n e p 1} & =\frac{2}{3} K H \times 1.1282 a_{\text {nec }}{ }^{-0.2544} a_{\text {nep } 1}^{1.2544} \\
a_{\text {nec }} & <a_{\text {nep } 1}<a_{\text {nepc }} \\
F_{\text {nep } 2} & =\frac{2}{3} K H \times 1.4988 a_{\text {nec }}-0.1021 a_{\text {nep } 2}^{1.1021} \\
a_{\text {nepc }} & <a_{\text {nep } 2} \leqslant a_{n p c} \\
a_{\text {nepc }} & =7.1197 a_{\text {nec }} \quad a_{n p c}=205.3827 a_{\text {nec }}
\end{aligned}
$$

式中, $a_{n e p c}$ 为弹塑性第一接触变形临界接触面积; $a_{n p c}$ 为弹塑性第二接触变形临界接触面积; $a_{n e p 1}$ 和 $a_{n e p 2}$ 分别为弹塑性第一、二接触变形的接触面积, 其表达式如下

$$
\begin{aligned}
& \frac{a_{\text {nep } 1}}{a_{\text {nec }}}=0.93\left(\frac{\omega_{n}}{\omega_{\text {nec }}}\right)^{1.136} \\
& \frac{a_{\text {nep } 2}}{a_{\text {nec }}}=0.94\left(\frac{\omega_{n}}{\omega_{\text {nec }}}\right)^{1.146}
\end{aligned}
$$

\section{6 微凸体弹塑性接触变形模型}

当尺度等级为 $n$ 的微凸体的变形量 $\omega_{n}$ 大于 $110 \omega_{\text {nec }}$ 时, 微凸体发生完全塑性接触变形, 此时单 个微凸体承受的法向接触载荷为

$$
\begin{gathered}
F_{n p}=K_{1} \sigma_{s} a_{n p} \\
a_{n p}=2 \pi R_{n} \omega_{n}
\end{gathered}
$$

式中, $K_{1}$ 为较软材料的硬度与屈服强度之比, $K_{1}=H / \sigma_{s}$

从上式可以看出, 完全塑性接触变形状态的单 个微凸体所受法向接触载荷和接触面积的 1 次方成 正比。

综合第 1.4 和 1.5 节可知 ${ }^{[13]}$, 对于尺度等级为 $n$ 的微凸体, 当 $n \leqslant n_{e c}$ 时微凸体只发生弹性接触变 
形; 而当 $n_{e c}<n \leqslant n_{e p c}$ 时微凸体可能发生弹性接触 变形和弹塑性第一接触变形; 当 $n_{e p c}<n \leqslant n_{p c}$ 时微 凸体可能发生弹性接触变形、弹塑性第一接触变形 和弹塑性第二接触变形; $n>n_{p c}$ 时微凸体可能发生 任意接触变形。

\section{7 结合面法向接触载荷}

为简化结合面法向接触载荷的计算, 采用文献 [13]中的计算方式, 各等级微凸体接触面积的分布 函数为

$$
\begin{gathered}
n_{n}(a)=Q n(a) \\
Q=\frac{a_{l}}{\sum_{n=n_{\min }}^{n_{\max }} a_{n l}} \quad a_{l}=\max \left(a_{n l}\right) \\
n(a)=\frac{D-1}{2} \phi^{\frac{3-D}{2}} a_{l}^{\frac{D-1}{2}} a^{\frac{-(1+D)}{2}} \\
\phi=5.454 \exp \left(\frac{1-D}{0.628}\right)+1.499
\end{gathered}
$$

需要说明的是 $a_{n l}$ 为尺度等级为 $n$ 的微凸体对 应的最大变形量下的接触面积, 而微凸体的最大变 形量在其对应等级下的自身高度范围内取值, 随着 微凸体接触载荷从零开始增大的过程中, 其所对应 的最大变形也会从零向微凸体的自身高度变化, 各 尺度等级微凸体在不同变形阶段接触面积分别按式 (14)、(18)、(19)和(21)进行计算。

结合面法向接触载荷为

$$
\begin{gathered}
F_{a l l}=F_{r e}+F_{r e p 1}+F_{r e p 2}+F_{r p} \\
F_{r e}=\sum_{n=n_{\min }}^{n_{e c}} \int_{0}^{a_{n l}} F_{n e} Q n(a) \mathrm{d} a+ \\
\sum_{n=n_{e c}+1}^{n_{\max }} \int_{0}^{a_{n e c}} F_{n e} Q n(a) \mathrm{d} a= \\
Q \frac{D-1}{4-D} \phi^{3-D / 2} \times \frac{16 \pi^{0.5} E G^{D-2} \ln (\gamma)^{\frac{1}{2}}}{3 L^{D-1}} \times \\
\sum_{n=n_{\min }}^{n_{e c}} a_{n l}^{\frac{3}{2}} \gamma^{n(D-1)}+\sum_{n=n_{e c}+1}^{n_{\max }} a_{n l}^{\frac{D-1}{2}} a_{n e c}{ }^{\frac{4-D}{2}} \gamma^{n(D-1)} \\
F_{r e p 1}=\sum_{n=n_{e c}+1}^{n_{e p c}} \int_{a_{n e c}}^{a_{n l}} F_{n e p 1} Q n(a) \mathrm{d} a+ \\
\sum_{n=n_{e p c}+1}^{n_{\max }} \int_{a_{n e c}}^{a_{n e p c}} F_{n e p 1} Q n(a) \mathrm{d} a= \\
Q \frac{D-1}{3} \phi^{3-D / 2} \frac{1.282 K H}{1.7544-0.5 D} \times
\end{gathered}
$$

$$
\begin{aligned}
& \left\{\sum_{n=n_{e c}+1}^{n_{e p c}} a_{n e c}^{-0.2544}\left(a_{n l}^{1.7544-0.5 D}-a_{n e c}^{1.7544-0.5 D}\right) a_{n l}^{\frac{D-1}{2}}+\right. \\
& \left.\sum_{n=n_{\text {epc }}+1}^{n_{\max }} a_{n e c}^{-0.2544}\left(a_{n e p c}^{1.7544-0.5 D}-a_{n e c}^{1.7544-0.5 D}\right) a_{n l}^{\frac{D-1}{2}}\right\} \\
& F_{r e p 2}=\sum_{n=n_{e p c}+1}^{n_{p c}} \int_{a_{n e c}}^{a_{n l}} F_{n e p 2} Q n(a) \mathrm{d} a+ \\
& \sum_{n=n_{e p c}+1}^{n_{\max }} \int_{a_{\text {nec }}}^{a_{n p c}} F_{n e p 2} Q n(a) \mathrm{d} a= \\
& Q \frac{D-1}{3} \phi^{3-D / 2} \frac{1.4988 K H}{1.6021-0.5 D} \times \\
& \left\{\sum_{n=n_{e p c}+1}^{n_{p c}} a_{n e c}^{-0.1021}\left(a_{n l}^{1.6021-0.5 D}-a_{n e p c}^{1.6021-0.5 D}\right) a_{n l}^{\frac{D-1}{2}}+\right. \\
& \left.\sum_{n=n_{e p c}+1}^{n_{\max }} a_{n e c}^{-0.1021}\left(a_{n p c}^{1.6021-0.5 D}-a_{n e p c}^{1.6021-0.5 D}\right) a_{n l}^{\frac{D-1}{2}}\right\} \\
& F_{r p}=\sum_{n=n_{p c}}^{n_{\max }} \int_{a_{n p c}}^{a_{n l}} F_{n p} Q n(a) \mathrm{d} a= \\
& Q \frac{D-1}{3-D} H \phi^{3-D / 2} \sum_{n=n_{p c}}^{n_{\max }}\left[a_{n l}^{\frac{3-D}{2}}-a_{n p c}{ }^{\frac{3-D}{2}}\right] a_{n l}^{\frac{D-1}{2}}
\end{aligned}
$$

式中, $n_{\min }$ 为结合面微凸体最小等级, 其与结合面 微凸体的平均高度有关，具体计算方法见第 3.2 节; $F_{r e}$ 代表结合面上处于弹性接触变形状态的微凸体 所承担的法向接触载荷之和, $F_{r e p 1}$ 代表结合面上处 于弹塑性第一接触变形状态的微凸体所承担的法向 接触载荷之和, $F_{\text {rep } 2}$ 代表结合面上处于弹塑性第二 接触变形状态的微凸体所承担的法向接触载荷之 和, $F_{r p}$ 代表结合面上处于完全塑性接触变形状态的 微凸体所承担的法向接触载荷之和。

\section{8 结合面静摩擦因数尺度关联三维分形模型}

根据第 1.3 节, 可得结合面上处于弹性接触变形 状态的微凸体和处于弹塑性第一接触变形状态的微 凸体所能承受的最大切向载荷分别为

$$
\begin{gathered}
T_{n e}=\frac{8 a_{n e} \sigma_{s}}{\pi(6-3 v)}+\frac{8(2 v-1)}{\pi(6-3 v)} F_{n e} \\
T_{n e p 1}=\frac{8 a_{n e p 1} \sigma_{s}}{\pi(6-3 v)}+\frac{8(2 v-1)}{\pi(6-3 v)} F_{n e p 1}
\end{gathered}
$$

那么, 结合面所能承受的最大切向载荷即最大 静摩擦力为

$$
T=T_{r e}+T_{r e p 1}
$$

式中, $T_{r e}$ 为结合面上处于弹性接触变形状态的微凸 体所能承担的最大切向载荷之和; $T_{r e p 1}$ 为结合面上 
处于弹塑性第一接触变形状态的微凸体所能承担的 最大切向载荷之和。

$T_{r e}=\sum_{n=n_{\min }}^{n_{e c}} \int_{0}^{a_{n l}} T_{n e} Q n(a) \mathrm{d} a+\sum_{n=n_{e c}+1}^{n_{\max }} \int_{0}^{a_{n e c}} T_{n e} Q n(a) \mathrm{d} a=$

$Q \phi^{\frac{3-D}{2}} \frac{D-1}{2}\left(\sum_{n=n_{\min }}^{n_{e c}}\left[\frac{2}{3-D} \frac{8 \sigma_{s}}{\pi(6-3 v)} a_{n l}+\right.\right.$

$\left.\frac{8(2 v-1)}{\pi(6-3 v)} \gamma^{n(D-1)} \frac{16 E \pi^{0.5} G^{D-2} \ln (\gamma)^{0.5}}{3 L^{D-1}} \frac{2}{4-D} a_{n l}^{1.5}\right]+$

$\sum_{n=n_{e c}+1}^{n_{\max }}\left[\frac{2}{3-D} \frac{8 \sigma_{s}}{\pi(6-3 v)} a_{n e c}^{\frac{3-D}{2}} a_{n l}^{\frac{D-1}{2}}+a_{n e c}^{\frac{4-D}{2}} \times\right.$

$\left.\left.\frac{8(2 v-1)}{\pi(6-3 v)} \gamma^{n(D-1)} \frac{16 E \pi^{0.5} G^{D-2} \ln (\gamma)^{0.5}}{3 L^{D-1}} a_{n l}^{\frac{D-1}{2}}\right]\right)$

$T_{r e p 1}=\sum_{n=n_{e c}+1}^{n_{e p c}} \int_{a_{\text {nec }}}^{a_{n l}} T_{n e p 1} Q n(a) \mathrm{d} a+$

$\sum_{n=n_{e p c}+1}^{n_{\max }} \int_{a_{\text {nec }}}^{a_{\text {nepc }}} T_{n e p 1} Q n(a) \mathrm{d} a=Q \phi^{\frac{3-D}{2}} \frac{D-1}{2} \times$

$\left(\sum_{n=n_{e c}+1}^{n_{e p c}}\left[\frac{2}{3-D} \frac{8 \sigma_{s}}{\pi(6-3 v)} a_{n l}+a_{n e c}^{-0.2544} \times\right.\right.$

$\frac{8(2 v-1)}{\pi(6-3 v)} \frac{2}{3} K H \times 1.1282 \frac{1}{1.7544-0.5 D} a_{n l}^{1.2544}$

$a_{n l}^{\frac{D-1}{2}} \frac{2}{3-D} \frac{8 \sigma_{s}}{\pi(6-3 v)} a_{n e c}{ }^{\frac{3-D}{2}}-\frac{8(2 v-1)}{\pi(6-3 v)} \frac{2}{3} K H \times$

$\left.\left.1.1282 a_{n e c}^{-0.2544} \frac{a_{n e c}^{1.7544-0.5 D}}{1.7544-0.5 D} a_{n l}^{\frac{D-1}{2}}\right]\right)+$

$Q \phi^{\frac{3-D}{2}} \frac{D-1}{2}\left(\sum_{n=n_{e p c}+1}^{n_{\max }}\left[\frac{2}{3-D} \frac{8 \sigma_{s}}{\pi(6-3 v)} a_{n e p c}{ }^{\frac{3-D}{2}} \times\right.\right.$

$a_{n l}^{\frac{D-1}{2}}+\frac{8(2 v-1)}{\pi(6-3 v)} \frac{2}{3} K H \times 1.1282 a_{n e c}^{-0.2544} \times$

$\frac{a_{n e p c}^{1.7544-0.5 D}}{1.7544-0.5 D} a_{n l}^{\frac{D-1}{2}}-\frac{2}{3-D} \frac{8 \sigma_{s}}{\pi(6-3 v)} a_{n e c}{ }^{\frac{3-D}{2}} \times$

$a_{n l}^{\frac{D-1}{2}}-\frac{8(2 v-1)}{\pi(6-3 v)} \frac{2}{3} K H \times 1.1282 a_{n e c}^{-0.2544} \times$

$\left.\left.\frac{a_{n e c}^{1.7544-0.5 D}}{1.7544-0.5 D} a_{n l}^{\frac{D-1}{2}}\right]\right)$

为了使比较的结果具有通用性, 这里将上述模 型中的参数进行量纲一化, 量纲一化的结合面法向 接触载荷为 $F^{*}=F_{\text {all }} /\left(E A_{a}\right)$, 量纲一化的结合面最 大切向载荷为 $T^{*}=T /\left(E A_{a}\right)$ 。

则结合面静摩擦因数为

$$
f=\frac{T^{*}}{F^{*}}
$$

以上所建模型即为结合面静摩擦因数尺度关联 三维分形模型，很明显它是与粗粘表面微凸体尺度 等级有关的, 这也是它与文献[1-3]模型的重要区别, 同时它是三维分形模型。

2 结合面静摩擦因数三维分形模型的 仿真与讨论

将文献[13]中的相关数据代入本文以上所建模 型, 运用数值仿真的方法, 观察在相同的特征尺度 系数下结合面分形维数与各变形阶段临界尺度等级 的关系和在分形维数为定值时结合面静摩擦因数随 结合面量纲一化法向接触载荷的变化规律, 并给出 不同分形维数下结合面弹性接触变形微凸体和结合 面弹塑性第一接触变形微凸体分别所能承受的最大 切向载荷占结合面所能承受的最大切向载荷的比值 关系。接触表面的材料性能参数 $E=52 \mathrm{GPa}$, 硬度为 $H=6.2 \mathrm{GPa}$, 泊松比 $v=0.3$, 特征尺度参数 $G=1.36 \times 10^{-7} \mathrm{~m}$, 微凸体的尺度等级范围取 $4 \sim 34^{[13]}$, 仿真结果如下图所示。

\section{1 微凸体临界尺度等级与分形维数之间的关系}

图 2 给出了微凸体临界尺度等级与结合面分形 维数之间的关系。从中可以看出, 随着分形维数由 小变大的过程中, 微凸体各个变形阶段临界尺度等 级都在增加, 且最后增幅均慢慢减缓, 其中微凸体 弹塑性第二接触变形临界尺度等级即 $n_{p c}$ 位于其他 接触变形临界尺度等级的最上方, 这与文献[15]中 的结论一致。

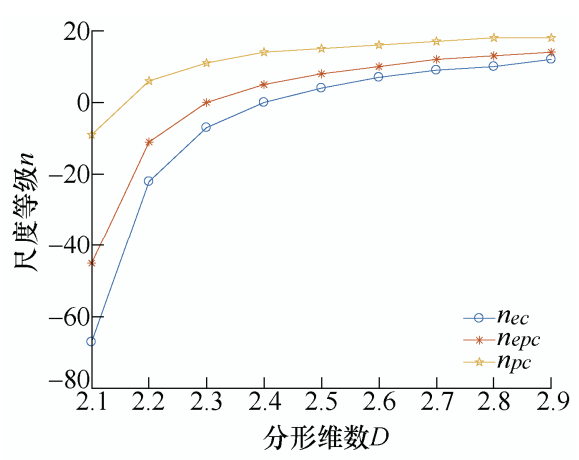

图 2 结合面分形维数与微凸体临界尺度等级的关系

\section{2 结合面静摩擦因数与量纲一化法向接触载荷 之间的关系}

图 3 中给出了在相同分形维数的条件下结合面 静摩擦因数 $f$ 随量纲一化法向接触载荷 $F^{*}$ 的变化 关系。 


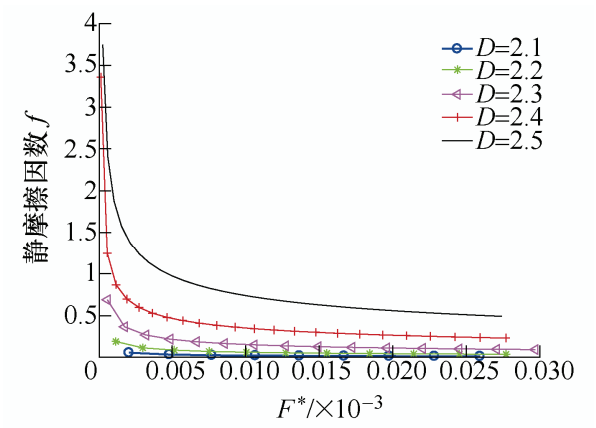

(a) 法向接触载荷

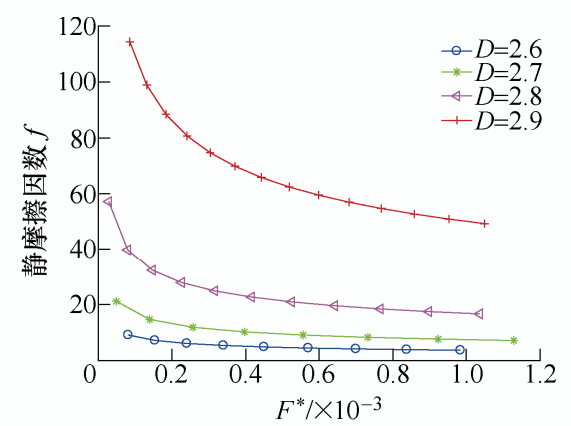

(b) 法向接触载荷

图 3 结合面静摩擦因数与量纲一化法向接触载荷的关系

由图 3 可知, 结合面静摩擦因数随着量纲一化 法向接触载荷的增大而呈凹弧式减小, 原因在于随
着结合面量纲一化法向接触载荷的增大, 越来越多 的微凸体由完全弹性接触变形和弹塑性第一接触变 形向弹塑性第二接触变形和完全塑性接触变形状态 转变, 而处于这两种变形状态的微凸体不再能够承 受切向载荷, 对结合面静摩擦力没有贡献。这一结 论与传统库仑摩擦定律认为静摩擦因数与法向载荷 的大小无关不同, 与文献[9-10]中的试验研究结果一 致, 说明本文所建模型是正确的。结合面静摩擦因 数随着结合面分形维数的增大而增大, 如图 3 所示。 由图 2 可以得到随着分形维数的增大, 各个接触变 形阶段临界等级变大, 而在同样的变形量下使得处 于完全弹性接触变形状态和第一弹塑性接触变形状 态的微凸体增多, 而只有处于这两种接触变形状态 的微凸体对结合面静摩擦力有贡献, 故而静结合面 摩擦因数随分形维数增大而增大。

\section{3 不同接触变形状态微凸体所能承受最大切向载 荷占比随量纲一化法向接触载荷之间的变化}

这里的不同接触变形状态是指结合面微凸体受 法向接触载荷作用处于弹性接触变形状态和弹塑性 第一接触变形状态。

图 4 所示为在法向接触载荷作用下结合面上分

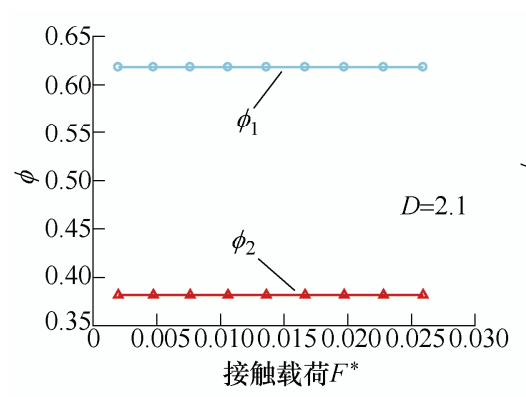

(a)

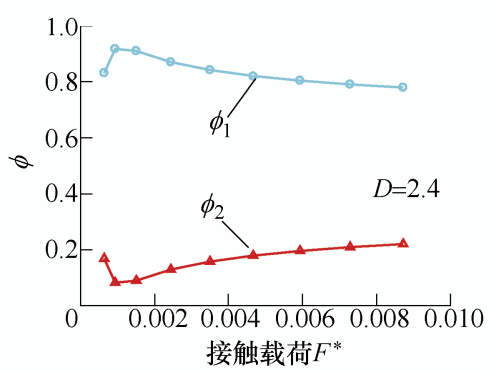

(d)

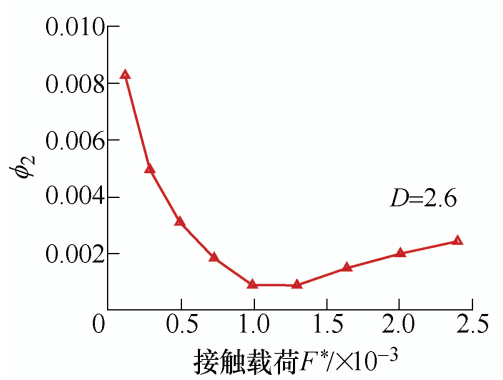

(g)

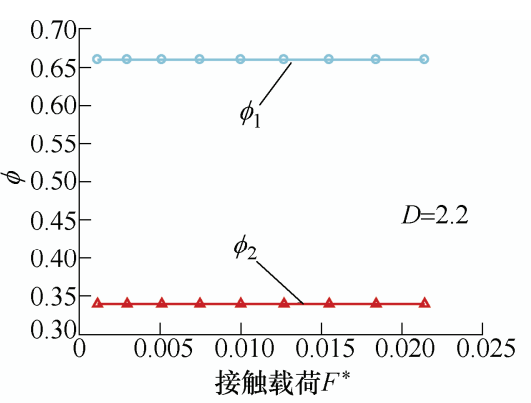

(b)

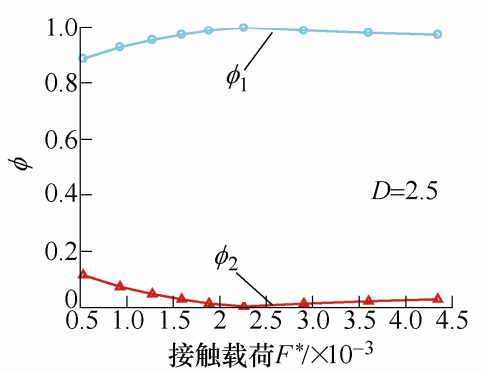

(e)

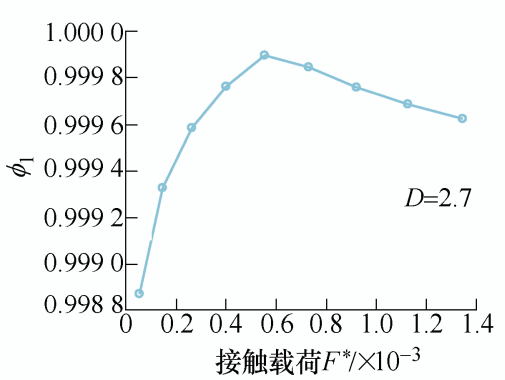

(h)

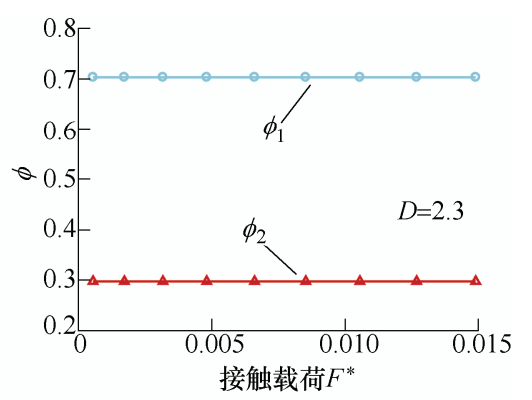

(c)

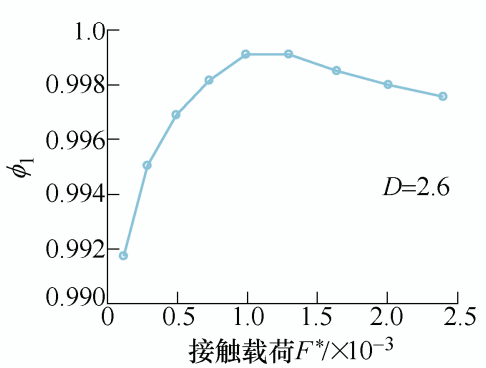

(f)

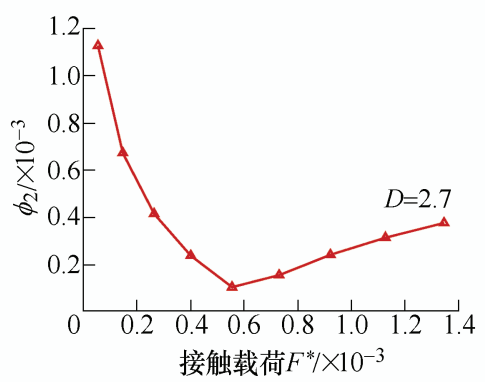

(i) 


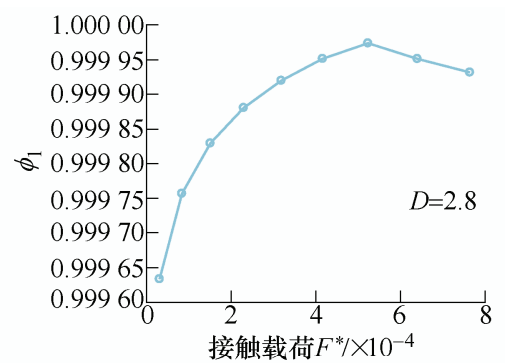

(j)

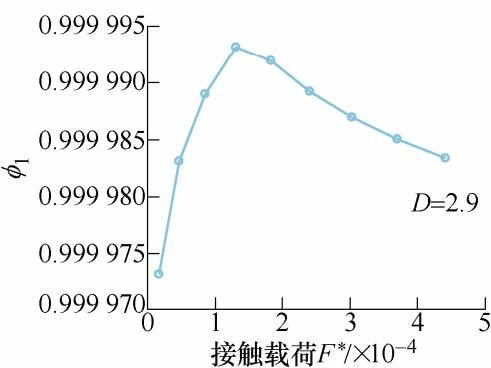

(1)

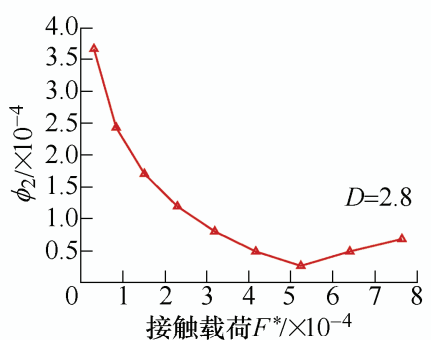

(k)

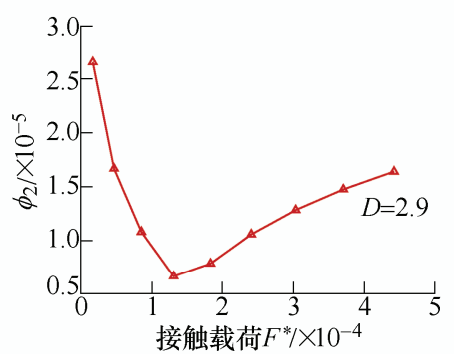

(m)

图 $4 \phi_{1} 、 \phi_{2}$ 随结合面无量纲法向接触载荷的变化规律

别处于弹性、弹塑性第一接触变形状态的微凸体分 别所能承受的最大切向载荷占结合面所能承受的最 大切向载荷的比值 $\phi_{1}=T_{r e} / T 、 \phi_{2}=T_{r e p 1} / T$ 随结合面量 纲一化法向接触载荷的变化规律。

因为当分形维数 $D>2.5$ 时, 在法向接触载荷的 作用下结合面上处于弹性和弹塑性第一接触变形状 态的微凸体分别所能承受的最大切向载荷占结合面 所能承受的最大切向载荷的比值 $\phi_{1}$ 与 $\phi_{2}$ 数量级相差 较大，故将二者分开表示以方便于清晰比较。

从图 4 中可以看出, 在结合面法向接触载荷较 小时, 结合面上弹性接触变形的微凸体对结合面静 摩擦力的贡献率较大, 特别是在分形维数 $D=2.9$ 时, 这一贡献率即占比 $\phi_{1}$ 几乎达 $100 \%$, 这是因为随着 分形维数的增高, 微凸体接触变形临界弹性等级升 高, 甚至会出现微凸体高度小于临界弹性变形量的 情况, 对应的发生弹性接触变形微凸体的概率会大 幅增加。随着法向载荷的增加, 这一贡献率变化方 向会发生突变, 在拐点后呈现出 $\phi_{1}$ 逐渐下降, 而 $\phi_{2}$ 逐渐升高, 但 $\phi_{1}$ 仍然占据主导地位, 说明微凸体的 弹性接触变形比弹塑性第一接触变形对结合面静摩 擦因数的影响大。需要注意的是分形维数较低时 $\phi_{1}$ 与 $\phi_{2}$ 的差别相比于分形维数高时小, 这是因为分 形维数越低, 微凸体接触变形临界弹性等级降低, 微凸体发生弹塑性第一接触变形的概率相比分形维 数较高时有所提高。

从图 4 可以看出, 随着分形维数的增大, $\phi_{1}$ 与 $\phi_{2}$ 变化的拐点减小, 这是因为随着分形维数增大, 结 合面变得光滑起来, 结合面微观几何形貌越来越精
细。由式(4)可知, 在分形特征长度尺度参数 $G$ 和微 凸体尺度等级 $n$ 一定时, 微凸体峰顶曲率半径随分 形维数的增大而减小，导致微凸体临界弹性接触变 形量减小, 由弹性接触变形转为弹塑性接触变形的 临界载荷减小。

\section{3 结合面静摩擦因数的验证与结果分析}

\section{1 不同模型的计算结果比较分析}

根据文献[13]可知, 粗䊁表面轮廓二维分形维 数 $D_{s}$ 、特征尺度系数 $G$ 与表面粗糙度 $R_{a}$ 之间的关 系为

$$
\begin{gathered}
D_{s}=\frac{1.54}{R_{a}^{0.042}} \\
G=10^{\frac{-5.26}{R_{a}^{0.042}}}
\end{gathered}
$$

文献[17]中采用三维接触轮廓仪测量了 17-4PH 不锈钢粗粗表面的参数，如图 5 所示。上部移动试

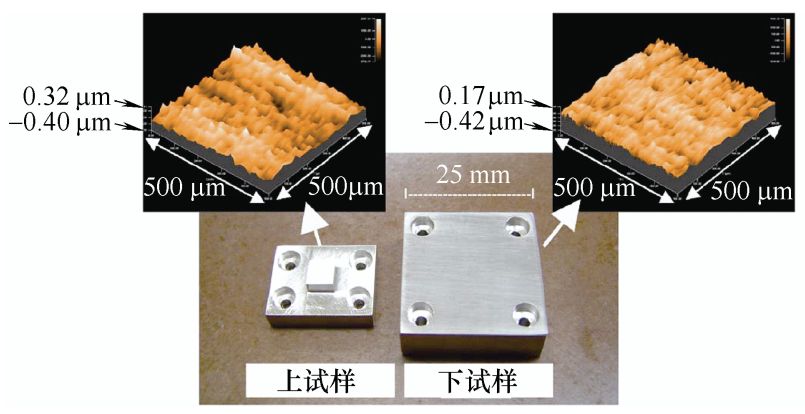

图 5 使用三维接触轮廓仪测量的典型试样和 表面粗粮度轮廓 ${ }^{[17]}$ 
样尺寸为 $5 \mathrm{~mm} \times 5 \mathrm{~mm}$, 下部试样尺寸为 $25 \mathrm{~mm} \times$ $25 \mathrm{~mm}$, 结合面名义接触面积为 $25.00 \mathrm{~mm}^{2}$ 。结合面 材料复合弹性模量为 $E=105.3 \mathrm{GPa}$, 硬度为 $H=2.96 \mathrm{GPa}$, 泊松比 $v=0.29$, 塑性指数为 1.04 。将 其代入经典 $\mathrm{KE}$ 模型可以计算获得结合面静摩擦因 数与法向载荷的关系。

为验证本文所建模型的合理性, 本文模型也采 用文献[17]中的试验数据对结合面静摩擦因数和法 向载荷进行计算, 由文献[17]采用三维接触轮廓仪 所测得的 $17-4 \mathrm{PH}$ 不锈钢试件上表面粗粘度 $R_{a 1}=0.024 \mu \mathrm{m}$, 下表面粗糙度 $R_{a 2}=0.021 \mu \mathrm{m}$, 根据 文献 [19] , 结合面综合粗粘度为 $R_{a}=\sqrt{{R_{a 1}{ }^{2}+R_{a 2}{ }^{2}}^{2}}=0.0316 \mu \mathrm{m}$, 将其代入式(34)和(35), 可得结合面三维分形参数 $D=D_{s}+1=2.78, G=8.287 \times$ $10^{-7}$, 进而将其代入本文所建模型并与文献[17]的计 算结果进行对比, 如图 6 所示。

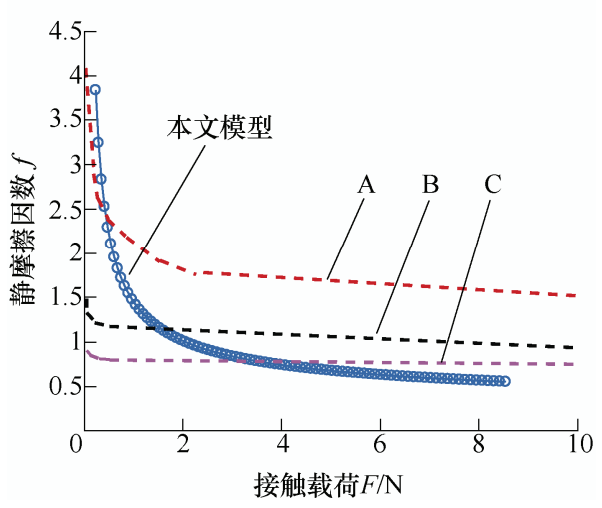

图 6 本文模型与 $\mathrm{KE}$ 模型对比图

图 6 中曲线 $A 、 B 、 C$ 在文献[17]中分别代表 结合面黏着力能为 $1.0 \mathrm{~N} / \mathrm{m} 、 0.5 \mathrm{~N} / \mathrm{m} 、 0.1 \mathrm{~N} / \mathrm{m}$ 时 采用经典 $\mathrm{KE}$ 模型计算得到的结合面静摩擦因数随 法向载荷的变化曲线图, 黏着力能参数是衡量结合 面间分子相互作用力强弱的物理量。在相同的法向 接触载荷条件下, 黏着力能参数越大说明两接触表 面间的分子吸引力越强 ${ }^{[17]}$, 其中横坐标即结合面法 向接触载荷最大 $10 \mathrm{~N}$ 是文献[17]提供的数据, 其结 合面名义接触面积仅为 $25.00 \mathrm{~mm}^{2}$ 。从图 6 可以看 出本文模型计算结果在较低载荷时大于考虑黏着 力能的 $\mathrm{KE}$ 模型, 这是因为结合面静摩擦因数尺度 关联三维分形模型利用尺度等级 $n$ 和分形参数能 够识别微凸体高度, 计算结合面静摩擦因数时其微 凸体高度取值在一个从小到大的范围内, 这一点与 传统统计静摩擦因数模型如 $\mathrm{KE}$ 模型不同。传统统 计模型在计算静摩擦因数时微凸体高度服从正态 分布, 其刻画微凸体高度时没有上限, 其取值可达
到正无穷, 这不符合结合面微凸体实际高度范 围 ${ }^{[18]}$, 因此在较低的法向接触载荷作用时其理论上 最先开始接触的极少部分正无穷高度微凸体其已 经发生塑性变形, 对结合面静摩擦因数没有贡献, 故而在小载荷时静摩擦因数偏小, 随着载荷增大到 一个范围时, 这一现象逐渐消失, 同时黏着力能影 响显现, 考虑黏着力能的静摩擦因数大于不考虑的 值。结合面黏着力能越大, 结合面静摩擦因数越 $大^{[17]}$ 。本文所建模型没有考虑结合面黏着力能, 所 以基本小于文献[17]中 $\mathrm{KE}$ 模型计算结果。从图 6 中可以看出本文所建模型的结合面静摩擦因数随 法向接触载荷的增大而减小, 这与文献[17]中 $\mathrm{KE}$ 模型的计算结果规律一致, 也与文献[1]CEB 摩擦 因数模型的结论相同, 同时也与文献 $[9-10]$ 的试验 研究结果结论规律一致, 说明了本文所建模型是正 确的。

\section{2 结合面静摩擦因数的试验实例验证}

文献[17]虽然测得了粗䊁表面的参数, 并将这 些数据代入 KE 静摩擦因数模型进行了仿真分析, 但并未对结合面法向接触载荷和静摩擦因数的关系 进行试验。为了进一步说明本文所建模型的正确性, 这里采用文献[19]中试验测得的结合面静摩擦因数 进一步进行验证。文献[19]对圆盘实际接触表面的 静摩擦因数进行了试验测定, 所测得的结合面参数 如下表 1 所示。

\section{表 1 文献[19]的结合面相关参数}

\begin{tabular}{lc}
\hline \multicolumn{1}{c}{ 参数 } & 数值 \\
\hline 结合面微凸体半径的取值范围 $R / \mu \mathrm{m}$ & $176.2 \sim 264.2$ \\
复合弹性模量 $E / \mathrm{GPa}$ & 59.52 \\
名义接触面积 $A_{n} / \mathrm{mm}^{2}$ & 78.54 \\
较软材料的硬度 $H / \mathrm{GPa}$ & 0.785 \\
\hline
\end{tabular}

结合面相关参数如表 1 中所示。事实上对于确 定的粗糙表面, 其所有微凸体半径不是一个固定值, 应该是在一定范围内变化。以 $\mathrm{GW}$ 模型为基础所建 立的结合面统计接触模型如文献[20]在最终计算时 都取半径为一固定值。结合面静摩擦因数尺度关联 三维分形模型可以解决了这一问题, 它利用尺度等 级可以刻画粗粘表面微凸体的不同大小及其半径, 更加符合实际情况。文献[19]取样长度取 $L=8.8 \mathrm{~mm}$ 。

由文献[21]可知, 对于确定的表面, 微凸体的 最大高度 $h$ 与粗䊁表面平均粗粘度 $R_{a}$ 之间满足以下 关系

$$
h=\frac{R_{a}}{k}
$$


式中, $k$ 为粗糙表面的完整系数, 且当粗粘表面配 对平面时 $k=1$, 文献[22-23]假设粗粘表面微凸体顶 端为球截状, 且其附着在基体上, 则粗糙表面微凸 体的最大高度 $h$ 可表述为微凸体半径的最大值即 $264.2 \mu \mathrm{m}$, 表面粗粘度 $R_{a}$ 为 $264.2 \times 10^{-6} \mathrm{~m}$, 将其代 入式 (34) 和 (35) 得粗䊅表面三维分形参数 $D=D_{s}+1=2.2185, G=6.8913 \times 10^{-5} \mathrm{~m}$, 粗䊁表 面微凸体的最大尺度等级应用

$$
n_{\max }=\operatorname{int}\left[\frac{\ln \left(L / L_{S}\right)}{\ln \gamma}\right]
$$

进行计算, 粗糙表面微凸体的最小尺度等级采 用式(5)进行反求解。由文献[24]可知 $\delta_{n \text { min }}$ 为微凸体 的平均高度, 由表 1 知微凸体的平均高度

$$
\delta_{n \min }=\frac{264.2+176.2}{2}=220.2 \mu \mathrm{m}
$$

这与文献[18]中计算微凸体平均半径的方法一 致, 将其代入式(5)可计算求得微凸体的最小尺度等 级。综上计算可得粗粘表面微凸体尺度等级取值范 围为 6 39。将其代入本文模型采用 Matlab 软件对 结合面静摩擦因数进行数值仿真计算, 并与文献[19] 统计模型(简称 ET 模型)、文献[25]统计模型(简称 $\mathrm{J}-\mathrm{M}$ 模型) 和试验测得的结合面静摩擦因数进行比 较, 结果如图 7 所示。

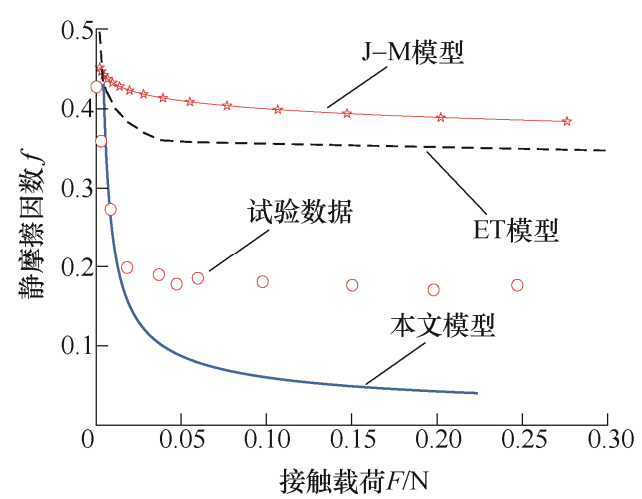

图 7 本文模型与试验数据对比图

从图 7 中可以看出本文所建模型的结合面静摩 擦因数随法向接触载荷的增大而减小, 并逐渐趋于 稳定, 这与文献[19]中试验结果规律一致, 也与文 献[19]和文献[25]静摩擦因数统计模型的结论相同, 同时也与文献[9-10]的试验研究结果规律一致, 说明 了本文所建模型是正确、合理的。图 7 中横坐标是结 合面法向接触载荷, 文献[19]给出的最大值为 $0.3 \mathrm{~N}$ 。 从图 6 中可以看出当法向接触载荷小于 $0.025 \mathrm{~N}$ 时 本文模型相比较于 $\mathrm{J}-\mathrm{M}$ 模型和 ET 模型与试验数据
拟合较好, 随着载荷的增大, 本文模型计算结果小 于试验结果数据, 这是因为本文模型未考虑微凸体 基体变形与微凸体相互作用。文献[23-26]研究表 明相同分形参数下考虑微凸体基体变形与微凸体 相互作用后结合面微凸体接触面积或变形量较不 考虑时偏小, 表现为结合面弹性变形和弹塑性第 一变形的微凸体所占比例增加, 而产生弹性变形 和弹塑性第一变形的微凸体对结合面静摩擦因数 有贡献，因此考虑微凸体基体变形与微凸体相互 作用后的结合面静摩擦因数将会增大, 也将更接 近于试验结果。

由上可见, 本文模型解决了现有结合面静摩擦 因数分形模型计算结果与试验结果趋势不一致的 问题。

\section{4 结论}

(1) 本文提出了一种尺度关联的结合面静摩擦 因数三维分形模型, 解决了现有结合面静摩擦因数 分形模型与静摩擦因数统计模型之间的矛盾, 即结 合面静摩擦因数随着结合面法向接触载荷的增大而 减小, 并与前人试验研究结果规律一致。

(2) 结合面静摩擦因数随着结合面分形维数增 大而增大。

\section{参 考 文 献}

[1] CHANG W R, ETSION I, BOGY D B . Static friction coefficient model for metallic rough surfaces[J]. Journal of Tribology, 1988, 110(1): 57-63.

[2] KOGUT L, ETSION I. A static friction model for elastic-plastic contacting rough[J]. Journal of Tribology, 2004, 126(1): 34-40.

[3] KOGUT L, ETSION I. A semi-analytical solution for the sliding inception of a spherical contact[J]. Journal of Tribology, 2003, 125(3): 499.

[4] YOU J M , CHEN T N. A static friction model for the contact of fractal surfaces[J]. Proceedings of the Institution of Mechanical Engineers, Part J: Journal of Engineering Tribology, 2010, 224(5): 513-518.

[5] 尤晋闽, 陈天宁. 结合面静摩擦系数的统计模型 [J]. 振 动与冲击, 2010, 29(12): 26-29.

YOU Jinmin, CHEN Tianning. Statistical type of static friction coefficient of joint surface[J]. Journal of Vibration and Shock, 2010, 29(12): 26-29.

[6] 盛选禹, 雒建斌, 温诗铸. 基于分形接触的静摩擦系数 
预测 [J]. 中国机械工程, 1998, 9(7): 16-18.

SHENG Xuan, YAN Jianbin, WEN Shizhu. Prediction of static friction coefficient based on fractal contact[J]. China Mechanical Engineering, 1998，9(7): 16-18.

[7] 田红亮, 赵春华, 方子帆, 等. 金属材料表面静摩擦学 特性的预测研究-理论模型 $[\mathrm{J}]$. 振动与冲击, 2013, 32(12): 40-44.

TIAN Hongliang, ZHAO Chunhua, FANG Zifan, et al. Prediction study of surface static tribological properties of metallic materials-theoretical model[J]. Journal of Vibration and Shock，2013，32(12): 40-44.

[8] 田红亮, 刘芙蓉, 赵春华, 等. 金属材料表面静摩擦学 特性的预测研究——试验佐证 $[\mathrm{J}]$. 振动与冲击, 2014, 33(1): 209-220.

TIAN Hongliang, LIU Furong, ZHAO Chunhua, et al. Prediction of surface static tribological properties of metallic materials_ experimental support[J]. Journal of Vibration and Shock, 2014, 33(1): 209-220.

[9] DUNKIN J E, KIM D E. Measurement of static friction coefficient between flat surfaces[J]. Wear, 1996, 193(2): 186-192.

[10] IBRAHIM D R D, JACKSON R L , FLOWERS G T. Measurements of the Static Friction Coefficient Between Tin Surfaces and Comparison to a Theoretical Model[J]. Journal of Tribology, 2011，133(3): 031408.

[11] 张学良. 机械结合面动态特性及应用 $[\mathrm{M}]$. 北京: 中国 科技出版社, 2002 .

ZHANG Xueliang. Dynamic characteristics and application of mechanical joint surface[M]. Beijing: China Science and Technology Press, 2002.

[12] YAN W , KOMVOPOULOS K. Contact analysis of elastic-plastic fractal surfaces[J]. Journal of Applied Physics, 1998, 84(7): 3617-3624.

[13] 陈建江, 原园, 成雨, 等. 尺度相关的分形结合面法向 接触刚度模型 $[\mathrm{J}]$. 机械工程学报, 2018, 54(21): 127-137.

CHEN Jianjiang, YUAN Yuan, CHENG Yu, et al. Scale-dependent fractal joint surface normal contact stiffness model[J]. Journal of Mechanical Engineering, 2018, 54(21): 127-137.

[14] KOGUT L, ETSION L. Elastic-plastic contact analysis of a sphere and rigid flat $[\mathrm{J}]$. Journal of Applied Mechanics, 2002, 69: 657-662.

[15] 成雨. 三维分形表面的接触性能研究[D]. 西安: 西安理 工大学, 2017.

CHENG Yu. Study on contact performance of three-dimensional fractal surface [D]. Xi'an : Xi'an University of Technology, 2017.

[16] LIOU J L , LIN J F , LIOU J L , et al. A modified fractal microcontact model developed for asperity heights with variable morphology parameters[J]. Wear, 2010, 268(1): 133-144.

[17] CHUL H L, ANDREAS A. Static friction experiments and verification of an improved elastic-plastic model including roughness effects[J]. Tribology, 2007, 129(4): 754-760

[18] 李玲, 王晶晶, 裴喜永, 等. 机械结合面接触刚度建模 新方法[J]. 机械工程学报, 2020，56(9)：162-169.

LI Ling, WANG Jingjing, PEI Xiyong, et al. A new method for modeling the contact stiffness of mechanical joints[J].Chinese Journal of Mechanical Engineering, 2020, 56(9): 162-169.

[19] ANDREAS A, POLYCARPOU I E. Comparison of the static friction subboundary lubrication model with experimental measurements on thin-film disks[J]. Tribology Transactions, 1998, 41(2).

[20] 肖会芳, 孙韵韵, 徐金梧. 刚度连续、单调且光滑变化 的粗粘界面法向弹塑性接触模型 $[\mathrm{J}]$. 中南大学学报, 2019, 50(6): 1343-1350.

XIAO Huifang, SUN Yunyun, XU Jinwu. Normal elastoplastic contact model of rough interface with continuous rigidity, monotonous and smooth variation $[\mathrm{J}]$.Journal of Central South University, 2019, 50(6): 1343-1350.

[21] GREENWOOD J A, TRIPP J H. The elastic contact of rough spheres[J]. J. Appl. Mech., 1967, 34(1): 153-0.

[22] GREENWOOD J A, WILLIAMSON J B P. Contact of nominally flat surfaces [J].Proceedings of the royal society of. London, 1966, 295: 300-319.

[23] 张伟, 张学良, 温淑花, 等. 考虑微凸体基体变形和相 互作用的结合面法向接触刚度模型 $[\mathrm{J}]$. 西安交通大学 学报, 2020, 54(6): 115-121.

ZHANG Wei, ZHANG Xueliang, WEN Shuhua, et al. The normal contact stiffness model of the joint surface considering the deformation and interaction of the asperity matrix[J]. Journal of Xi'an Jiaotong University, 2020, 54(6): $115-121$.

[24] YUAN Y , CHENG Y , LIU K , et al. A revised Majumdar and Bushan model of elastoplastic contact between rough surfaces[J]. Applied Surface Science, 2017: S0169433217319499.

[25]YOU J M, CHEN T N. Statistical model for normal and tangential contact parameters of rough surfaces[J]. 
Proceedings of the Institution of Mechanical Engineers, 2011, 225(1): 1-15.

[26] 田小龙. 考虑微凸体相互作用的机械结合面接触刚度 模型[D]. 西安: 西安理工大学, 2015.

TIAN Xiaolong. Contact stiffness model of mechanical interface considering the interaction of micro convex bodies [D]. Xi'an: Xi'an University of Technology, 2015.

作者简介: 张学良, 男, 1964 年出生, 博士, 教授, 博士研究生导师。 主要研究方向为机械结合面接触特性、智能优化算法及应用、机电系统 结构动态特性与测试技术。

E-mail: zhang_xue_1@sina.com

张伟, 男, 1993 年出生。主要研究方向为机械结合面接触特性。

E-mail: S20180246@stu.tyust.edu.cn 